\title{
Effectiveness of administering zinc acetate hydrate to patients with inflammatory bowel disease and zinc deficiency: a retrospective observational two-center study
}

\author{
Kensuke Sakurai ${ }^{1}$, Shigeru Furukawa ${ }^{2}$, Takehiko Katsurada ${ }^{1}$, Shinsuke Otagiri ${ }^{1}$, Kana Yamanashi $^{3}$, Kazunori Nagashima $^{1}$ \\ Reizo Onishi ${ }^{1}$, Keiji Yagisawa ${ }^{2}$, Haruto Nishimura ${ }^{2}$, Takahiro Ito ${ }^{2}$, Atsuo Maemoto ${ }^{2}$, Naoya Sakamoto ${ }^{1}$ \\ ${ }^{1}$ Department of Gastroenterology and Hepatology, Hokkaido University Hospital, Sapporo; ${ }^{2}$ Inflammatory Bowel Disease Center, Sapporo \\ Higashi Tokushukai Hospital, Sapporo; ${ }^{3}$ Department of Gastroenterology and Hepatology, Sapporo Hokushin Hospital, Sapporo, Japan
}

Background/Aims: Inflammatory bowel disease (IBD) patients frequently have zinc deficiency. IBD patients with zinc deficiency have higher risks of IBD-related hospitalization, complications, and requiring surgery. This study aimed to examine the effectiveness of zinc acetate hydrate (ZAH; Nobelzin) in IBD patients with zinc deficiency. Methods: IBD patients with zinc deficiency who received ZAH from March 2017 to April 2020 were registered in this 2-center, retrospective, observational study. Changes in serum zinc levels and disease activity (Crohn's Disease Activity Index [CDAI]) before and after ZAH administration were analyzed. Results: Fifty-one patients with Crohn's disease $(C D, n=40)$ or ulcerative colitis $(U C, n=11)$ were registered. Median serum zinc level and median CDAI scores significantly improved $(55.5-91.0 \mu \mathrm{g} / \mathrm{dL}, P<0.001 ; 171.5-129, P<0.001$, respectively) in CD patients 4 weeks after starting ZAH administration. Similarly, median serum zinc levels and CDAI scores significantly improved $(57.0-81.0 \mu \mathrm{g} / \mathrm{dL}, P<0.001 ; 177-148, P=0.012$, respectively) 20 weeks after starting ZAH administration. Similar investigations were conducted in groups where no treatment change, other than ZAH administration, was implemented; significant improvements were observed in both serum zinc level and CDAI scores. Median serum zinc levels in UC patients 4 weeks after starting ZAH administration significantly improved from 63.0 to $94.0 \mu \mathrm{g} / \mathrm{dL}(P=0.002)$, but no significant changes in disease activity were observed. One patient experienced side effects of abdominal discomfort and nausea. Conclusions: ZAH administration is effective in improving zinc deficiency and may contribute to improving disease activity in IBD. (Intest Res 2022;20:78-89)

Key Words: Inflammatory bowel diseases; Zinc deficiency; Ulcerative colitis; Crohn disease; Zinc acetate

\section{INTRODUCTION}

Previous studies have indicated that inflammatory bowel disease (IBD) is generally associated with weight loss and malnutrition. ${ }^{1-4}$ Micronutrient deficiency is frequently observed in patients with IBD and has been associated with various symptoms, including anemia (deficiencies of iron, folate, and vita-

Received October 7, 2020. Revised December 122020.

Accepted December 19, 2020.

Correspondence to Takehiko Katsurada, Department of Gastroenterology

and Hepatology, Hokkaido University Hospital, Kita-15, Nishi-7 Kita-ku,

Sapporo 060-8648, Japan. Tel: +81-11-716-1161, Fax: +81-11-706-7999,

E-mail: tkatsu@amber.plala.or.jp min $B_{12}$ ), bone disease (deficiencies of Ca and vitamin $D$ ), hypercoagulable state (deficiencies of folate and vitamins $\mathrm{B}_{6}$ and $B_{12}$ ), and delays in wound healing (deficiencies of zinc and vitamins A and C). ${ }^{5}$ Given this, guidelines by the British Society of Gastroenterology recommend regular screening for micronutrient deficiency in IBD patients. ${ }^{6}$

Zinc is a micronutrient that plays a role in many functions, such as reproduction, bone growth, regulating taste responses, and immunity. ${ }^{7}$ Compared to healthy people, those with IBD tend to have zinc deficiency, ${ }^{8-10}$ the frequencies of which are very high: $42.2 \%$ (326/773) in Crohn's disease (CD) and 38.6\% (86/223) in ulcerative colitis (UC). ${ }^{11}$ It is considered that the 
causes of zinc deficiency in IBD patients mainly include low zinc intake, reduced zinc absorption, and chronic inflammation-induced hypoalbuminemia. ${ }^{10,12-14}$

IBD patients with zinc deficiency have a higher risk of surgery, hospitalization, and complications related to IBD than those without zinc deficiency. ${ }^{11}$ Moreover, a comparison between IBD patients with persistent zinc deficiency and those with zinc deficiency but zinc levels returned to normal within 12 months revealed that the risks of surgery, hospitalization, and complications related to IBD decreased in the latter. ${ }^{11}$

In 2017, the use of a zinc acetate hydrate (ZAH; Nobelzin ${ }^{\circledR}$ ) agent was approved by the Japanese National Health Insurance for zinc replacement therapy in patients with zinc deficiency. Therefore, an increasing number of IBD patients with zinc deficiency have received zinc replacement therapy in Japan.

However, to the best of our knowledge, no studies have examined the effectiveness of proactive zinc administration in IBD patients with zinc deficiency. This study aimed to examine the effectiveness of ZAH in IBD patients with zinc deficiency.

\section{METHODS}

\section{Study Design and Aims}

This two-center, retrospective, observational study was conducted at the Hokkaido University Hospital and Sapporo Higashi Tokushukai Hospital. The observation period was from March 2017 to April 2020. The period during which subjects began ZAH administration ranged from October 25, 2017 to August 31, 2019.

\section{Definition of Zinc Deficiency}

To ensure effective functioning of zinc in the human body, a serum zinc level of $\geq 80 \mu \mathrm{g} / \mathrm{dL}$ is recommended; the Japanese Society of Clinical Nutrition recommends zinc replacement therapy for patients with a serum zinc level below $80 \mu \mathrm{g} / \mathrm{dL} .^{15}$ Hence, this study defined a serum zinc level of $\geq 80 \mu \mathrm{g} / \mathrm{dL}$ as normal and $<80 \mu \mathrm{g} / \mathrm{dL}$ as deficient.

\section{Participants}

The patients are as follows: (1) patients who were diagnosed with either UC or CD based on endoscopy or pathological diagnostic criteria, ${ }^{16}(2)$ IBD patients who received ZAH for zinc deficiency during the period from March 2017 to April 2020; or (3) patients who underwent at least one follow-up after starting zinc administration and whose serum zinc level and disease activity were evaluated.

\section{Outcomes}

The normalization rate of zinc during the observation period and the factors contributing to the lack of improvement were analyzed. Changes in the serum zinc levels, disease activity, and hematological findings before and after ZAH administration (comparison between the administration start date and either 4 or 20 weeks after starting administration), and the correlations between serum zinc level and disease activity or C-reactive protein (CRP) were examined. Observations up to 8 weeks after administration were conducted for patients whose serum zinc level was not measured 4 weeks after administration; the data on the measurement date closest to 4 weeks after administration were used. Regarding the assessment at 20 weeks after administration, when observations were conducted up to 24 weeks after administration, the data on the measurement date closest to 20 weeks after administration were used. Disease activity was assessed using the partial Mayo score and Crohn's Disease Activity Index (CDAI) score for UC patients and CD patients, respectively. ${ }^{17-19}$

\section{Statistical Analysis}

The GraphPad Prism 8 software package (GraphPad Software Inc., San Diego, CA, USA) was used for all statistical analyses. All variables were expressed as a median (range) or number (\%). All reported $P$-values are two-sided; $P<0.05$ was considered statistically significant. $P$-values were calculated using the chi-squared test for categorical variables and Mann Whitney test or Wilcoxon matched-pairs signed-rank test for continuous variables. Correlations between serum zinc level, CDAI, and CRP were calculated using Spearman's correlation coefficient.

\section{Ethical Considerations}

This study commenced after obtaining approval from the human research ethics committees of Hokkaido University Hospital (IRB No. 019-0241) and Sapporo Higashi Tokushukai Hospital (IRB No. TGE01301-012). The study protocol was posted on the websites of all the study sites. Patients opted out from the study if they did not wish to give consent. The informed consent was waived.

\section{RESULTS}

\section{Patient Registration}

Between March 2017 and April 2020, serum zinc levels were measured in 482 IBD patients, of whom 276 were CD and 206 
were UC. Among them, 238 (238/276, 86.2\%) of CD patients had zinc deficiency $(<80 \mu \mathrm{g} / \mathrm{dL})$, and $103(103 / 276,37.3 \%)$ had serum zinc levels of $<60 \mu \mathrm{g} / \mathrm{dL}$. Zinc deficiency $(<80 \mu \mathrm{g} / \mathrm{dL})$ was observed in 140 UC patients (140/206, 68.0\%), and serum zinc level was $<60 \mu \mathrm{g} / \mathrm{dL}$ in 49 cases $(49 / 206,23.8 \%)$. A total of 56 IBD patients who received ZAH for zinc deficiency were registered for this study. Of these, the following patients were excluded: 4 patients who chose to discontinue the oral administration of the drug and 1 UC patient who underwent total proctocolectomy. The remaining 51 patients were enrolled. Of these, 32 CD patients and 9 UC patients whose serum zinc levels were measured 4 weeks after ZAH administration, and 25 CD patients and 5 UC patients whose serum zinc levels were measured 20 weeks after ZAH administration were finally included in the analysis (Fig. 1).

\section{CD Patients}

\section{1) Patient Background}

The median age of the CD patients was 39.5 years (range, 2363 years). This group consisted of 29 men (72.5\%) and 11 women $(27.5 \%)$ with a median duration of disease of 18 years (range, $0-32$ years). The disease affected the small intestine in 6 patients, the large intestine in 4 patients, and both the small and large intestine in 30 patients. The median CDAI score was

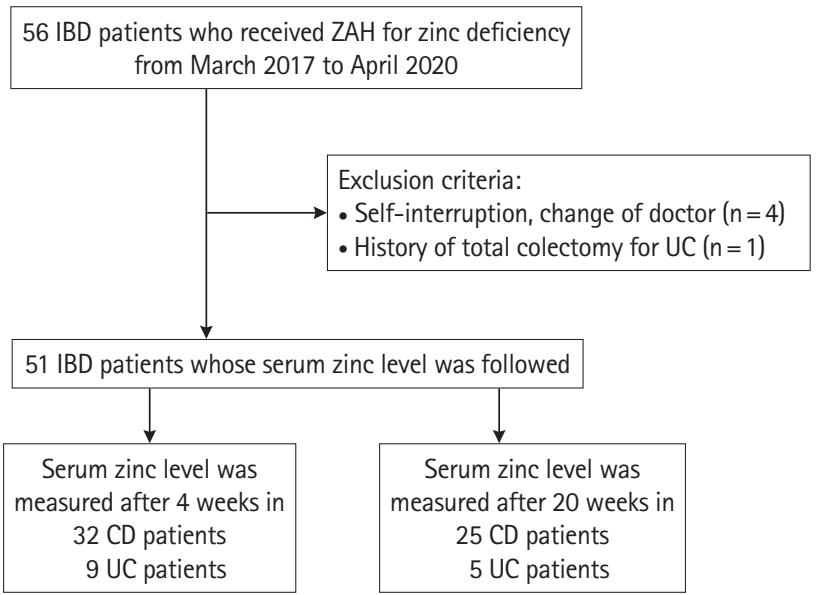

Fig. 1. Patient enrollment. Of the 56 inflammatory bowel disease (IBD) patients who were administered zinc acetate hydrate (ZAH) for zinc deficiency from March 2017 to April 2020, patients who self-interrupted administration, changed hospitals, or underwent total colectomy for ulcerative colitis (UC) were excluded; a total of 51 patients were included. Of these, analyses were conducted on 32 Crohn's disease (CD) patients and 9 UC patients whose serum zinc levels were measured 4 weeks after $\mathrm{ZAH}$ administration, and 25 CD patients and 5 UC patients whose serum zinc levels were measured 20 weeks after $\mathrm{ZAH}$ administration.
175 (range, 18-546), the median serum zinc level was $57.5 \mu \mathrm{g} /$ $\mathrm{dL}$ (range, $31-74 \mu \mathrm{g} / \mathrm{dL}$ ), and the median ZAH dose was 50 $\mathrm{mg} /$ day (range, 25-150 mg/day). Regarding concomitantly administered drugs, 25 patients $(62.5 \%)$ received enteral nutrition, 28 patients $(70 \%)$ received 5 -aminosalicylic acid/salazosulfapyridine, 10 patients (25\%) received corticosteroids, 21 patients (52.5\%) received immunomodulators, 20 patients (50\%) received anti-tumor necrosis factor (TNF)- $\alpha$ monoclonal antibodies, and 12 patients (30\%) received anti-interleukin

Table 1. Patients' Characteristics

\begin{tabular}{|c|c|c|}
\hline Characteristics & $C D(n=40)$ & $U C(n=11)$ \\
\hline Age (yr) & $39.5(23-63)$ & $56.0(28-87)$ \\
\hline \multicolumn{3}{|l|}{ Sex } \\
\hline Male & $29(72.5)$ & $7(63.6)$ \\
\hline Female & $11(27.5)$ & $4(36.3)$ \\
\hline Medical history (yr) & $18(0-32)$ & $8(0-47)$ \\
\hline \multicolumn{3}{|l|}{ Smoking status } \\
\hline Current & $12(30.0)$ & $2(18.2)$ \\
\hline Former & $5(12.5)$ & $2(18.2)$ \\
\hline Never & $23(57.5)$ & $7(63.6)$ \\
\hline \multicolumn{3}{|l|}{ Disease type } \\
\hline CD: ileitis/colitis/ileo-colitis & $\begin{array}{c}6(15.0) / 4(10.0) / \\
30(75.0)\end{array}$ & \\
\hline UC: proctitis/left side/pancolitis & & $\begin{array}{c}0(0) / 2(18.2) / \\
9(81.8)\end{array}$ \\
\hline \multicolumn{3}{|l|}{ Activity index } \\
\hline CDAl & $175(18-546)$ & \\
\hline pMayo & & $3(0-9)$ \\
\hline Serum zinc concentration ( $\mu \mathrm{g} / \mathrm{dL})$ & $57.5(31-74)$ & $63.0(46-74)$ \\
\hline Dose of ZAH (mg/day) & $50(25-150)$ & $100(25-100)$ \\
\hline \multicolumn{3}{|l|}{ Concomitant medication } \\
\hline Enteral nutrition & $25(62.5)$ & 0 \\
\hline $5-A S A, S A S P$ & $28(70.0)$ & $10(90.9)$ \\
\hline Corticosteroid & $10(25.0)$ & $2(18.1)$ \\
\hline Immunomodulator & $21(52.5)$ & $5(45.4)$ \\
\hline Calcineurin inhibitor & 0 & 0 \\
\hline Anti-TNF- $\alpha$ & $20(50.0)$ & $2(18.1)$ \\
\hline Anti-integrin & 0 & $1(9.1)$ \\
\hline Anti-IL-12/-23 & $12(30.0)$ & 0 \\
\hline JAK inhibitor & 0 & 0 \\
\hline
\end{tabular}

Values are presented as median (range) or number (\%).

CD, Crohn's disease; UC, ulcerative colitis; CDAl, Crohn's Disease Activity Index; pMayo, partial Mayo score; ZAH, zinc acetate hydrate; 5-ASA, 5-aminosalicylic acid; SASP, salazosulfapyridine; TNF, tumor necrosis factor; IL, interleukin; JAK, Janus kinase. 
(IL)-12/-23p40 antibodies (Table 1). In addition, as symptoms associated with zinc deficiency, dysgeusia was observed in 1 case, dermatitis in 3 cases, and stomatitis in 1 case.

\section{2) Clinical Results}

There were 32 CD patients whose serum zinc levels were measured 4 weeks after starting ZAH administration. The median serum zinc levels before and after ZAH administration for 4 weeks improved significantly from 55.5 to $91.0 \mu \mathrm{g} / \mathrm{dL}$ $(P<0.001)$, and serum zinc levels normalized in $71.9 \%$ of patients (23/32) $(\mathrm{Zn} \geq 80 \mu \mathrm{g} / \mathrm{dL})$. Furthermore, the median CDAI scores before and after ZAH administration significantly improved from 171.5 to $129(P<0.001)$ (Fig. 2A and B). Looking at the changes in the CDAI score for each subscore, the number of liquid/soft stools in the past 1 week, the sum of 7 daily abdominal pain ratings, the sum of 7 daily well-being ratings, and bodyweight percent below standard weight were significantly improved (Supplementary Table 1).

In the same group, $81.3 \%$ of CD patients (26/32) did not undergo any treatment change other than ZAH from 4 weeks before to 4 weeks after starting ZAH administration. The median serum zinc level before and after ZAH administration in the same group significantly increased from 57.0 to $91.0 \mu \mathrm{g} / \mathrm{dL}$ $(P<0.001)$, and serum zinc levels normalized in $76.9 \%$ of patients (20/26). Furthermore, the median CDAI scores before and after ZAH administration significantly improved from 145 to $116(P=0.003)$ (Fig. 3A and B). There were 3 cases in which the treatment was changed from 4 weeks before the start of ZAH administration to the start of administration. Double doses of adalimumab, infliximab, and enteral nutrition were introduced, respectively. There were 3 cases in which the treatment was changed from the start of ZAH administration to 4 weeks after administration. Corticosteroids were started in 3 cases, and ustekinumab was introduced in 2 cases.

There were no notable changes in other blood sampling items 4 weeks after ZAH administration (Supplementary Table 2).

There were 25 patients whose serum zinc levels were measured 20 weeks after starting ZAH administration. The median serum zinc levels before and after ZAH administration for 20 weeks improved significantly from 57.0 to $81.0 \mu \mathrm{g} / \mathrm{dL}(P<0.001)$, and serum zinc levels normalized in $52.0 \%$ of patients $(13 / 25)$. Furthermore, the median CDAI scores before and after ZAH administration significantly improved from 177 to $148(P=$ 0.012) (Fig. 2C and D). Looking at the changes in the CDAI score for each subscore, the sum of 7 daily well-being ratings
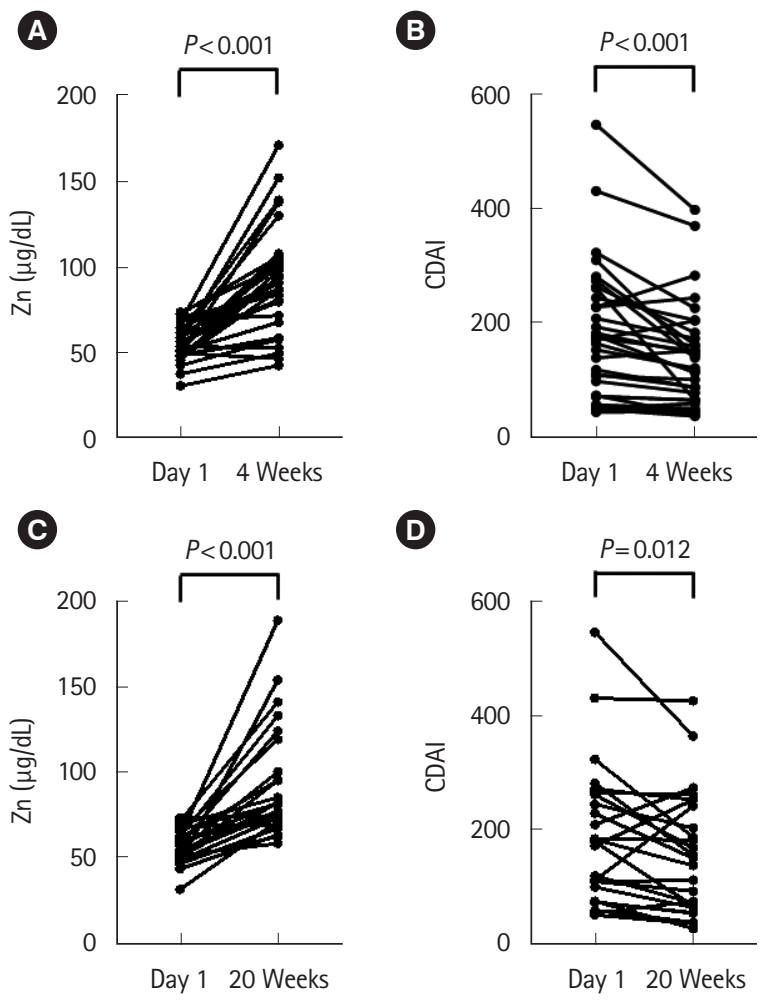

Fig. 2. Changes in serum zinc levels and Crohn's Disease Activity Index (CDAl) scores in Crohn's disease (CD) patients before zinc acetate hydrate (ZAH) administration and 4 weeks or 20 weeks after administration. $(A, B)$ The serum zinc levels of $32 \mathrm{CD}$ patients were measured 4 weeks after $\mathrm{ZAH}$ administration. Median serum zinc levels significantly improved from $55.5 \mu \mathrm{g} / \mathrm{dL}$ (31-74) to $91.0 \mu \mathrm{g} / \mathrm{dL}(43-171)(P<0.001)$. Median CDAl scores significantly improved from $171.5(43-546)$ to $129(36-397)(P<0.001)$. $(C, D)$ There were $25 \mathrm{CD}$ patients whose serum zinc levels were measured 20 weeks after ZAH administration. Median serum zinc levels significantly improved from $57.0 \mu \mathrm{g} / \mathrm{dL}(31-73)$ to $81.0 \mu \mathrm{g} / \mathrm{dL}$ (58-189) $(P<0.001)$. Median CDAl scores significantly improved from $177(43-546)$ to $148(36-397)(P=0.012)$.

were significantly improved (Supplementary Table 1).

In the same group, $76.0 \%$ of CD patients (19/25) did not undergo any treatment change other than ZAH from 4 weeks before to 20 weeks after starting ZAH administration. The median serum zinc level before and after ZAH administration in the same group significantly improved from 59.0 to $85.0 \mu \mathrm{g} /$ $\mathrm{dL}(P<0.001)$, and serum zinc levels normalized in $63.2 \%$ of patients (12/19). Furthermore, the median CDAI scores before and after ZAH administration significantly improved from 170 to $109(P=0.004)$ (Fig. 3C and D). There was 1 case in which the treatment was changed from 4 weeks before the start of ZAH administration to the start of the administration, and a double dose of adalimumab was administered. There 

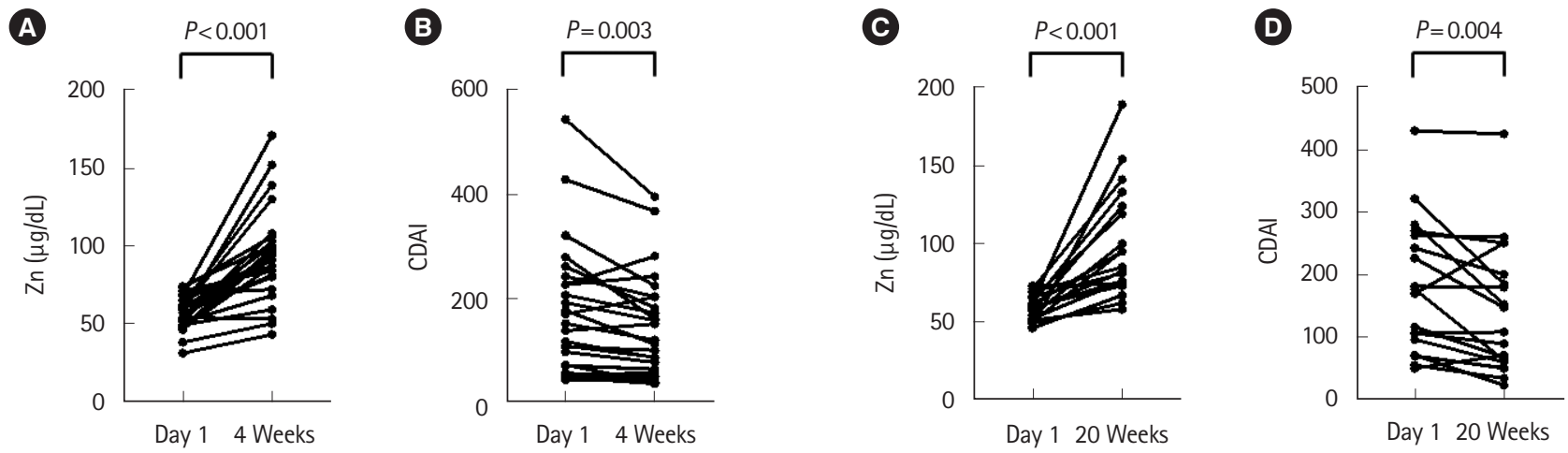

Fig. 3. Changes in serum zinc levels and Crohn's Disease Activity Index (CDAl) scores before and after zinc acetate hydrate (ZAH) administration in Crohn's disease (CD) patients, in whom changes in treatment other than ZAH were not implemented from 4 weeks before to 4 weeks or 20 weeks after starting ZAH administration. (A, B) A total of 81.3\% (26/32) of the CD patients did not undergo changes in treatment other than ZAH from 4 weeks before to 4 weeks after starting ZAH administration. The median serum zinc levels significantly improved from $57.0 \mu \mathrm{g} / \mathrm{dL}(31-74)$ to $91.0 \mu \mathrm{g} / \mathrm{dL}$ (43-171) $(P<0.001)$. Median CDAl scores significantly improved from 145 (43-546) to 116 (36-397) $(P=0.003)$. (C, D) A total of 76.0\% (19/25) of CD patients did not undergo changes in treatment other than ZAH from 4 weeks before to 20 weeks after starting ZAH administration. The median serum zinc levels significantly improved from $59.0 \mu \mathrm{g} / \mathrm{dL}$ (46-73) to $85.0 \mu \mathrm{g} / \mathrm{dL}(58-189)(P<0.001)$. Median CDAl scores significantly improved from $170(21-430)$ to 109 (21-425) $(P=0.004)$.
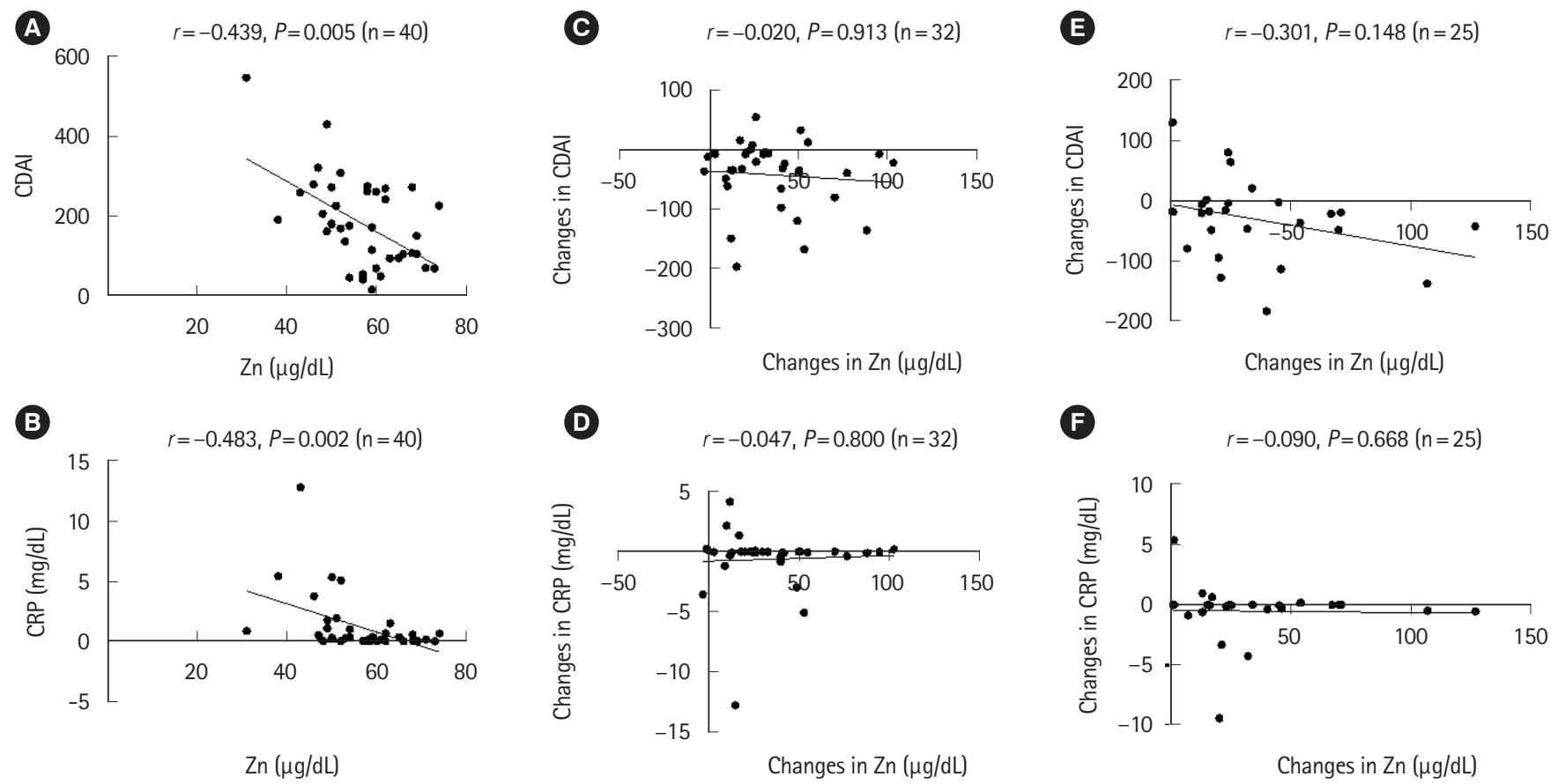

Fig. 4. Correlation between serum zinc level and Crohn's Disease Activity Index (CDAl) score or C-reactive protein (CRP) in Crohn's disease (CD) patients. (A, B) Before zinc acetate hydrate (ZAH) administration, there was a moderate correlation between serum zinc level and $\operatorname{CDAl}$ score $(r=-0.439, P=0.005)$, and there was a moderate correlation between serum zinc level and $\operatorname{CRP}(r=-0.483, P=0.002)$. (C, D) Four weeks after ZAH administration, there was no correlation between the degree of increase in serum zinc level and the degree of improvement in CDAl score $(r=-0.020, P=0.913)$, and there was no correlation between the degree of increase in serum zinc level and the degree of improvement in CRP $(r=-0.047, P=0.800)$. ( $E, F)$ Twenty weeks after ZAH administration, there was no correlation between the degree of increase in serum zinc level and the degree of improvement in CDAl score $(r=-0.301, P=0.148)$, and there was no correlation between the degree of increase in serum zinc level and the degree of improvement in CRP $(r=-0.090, P=0.668)$. 
were 5 cases in which treatment was changed between the start of ZAH administration and 20 weeks after administration. Corticosteroids were started in 3 cases, ustekinumab was introduced in 1 case, adalimumab was introduced in 1 case, azathioprine was introduced in 2 cases, enteral nutrition was introduced in 1 case, and home parenteral nutrition was introduced in 1 case.

There were no notable changes in other blood sampling items 20 weeks after ZAH administration (Supplementary Table 2).

The analysis was performed on the correlation between serum zinc level and CDAI score or CRP. There was a moderate correlation between serum zinc level and CDAI score before ZAH administration $(r=-0.439, P=0.005)$, and a moderate correlation between serum zinc level and CRP before ZAH administration $(r=-0.483, P=0.002)$ (Fig. $4 \mathrm{~A}$ and B). On the other hand, 4 weeks after ZAH administration, no significant correlation was demonstrated between the degree of increase in serum zinc level and the degree of improvement in CDAI score or CRP (Fig. 4C and D). Similarly, 20 weeks after ZAH administration, no significant correlation was demonstrated between the degree of increase in serum zinc level and the degree of improvement in CDAI score or CRP (Fig. 4E and F).

We also analyzed factors involved in the lack of serum zinc level normalization even after ZAH administration. Each item was compared by dividing the CD patients 4 weeks or 20 weeks after ZAH administration between the zinc normalization group and non-normalization group. Naturally, the rate of increase from the serum zinc level baseline was significantly higher in the zinc normalization group during both periods. In the group observed 4 weeks after ZAH administration, CRP before administration was significantly higher $(P=0.002)$, and serum zinc level before administration was lower $(P=0.007)$ in the zinc-normalized group. The concomitant rate of corticosteroid was significantly higher in the zinc-normalized group $(P=0.041)$ (Table 2). On the other hand, 20 weeks after ZAH administration, enteral nutrition was performed at a significantly higher frequency in the zinc-normalized group $(P=0.022)$ (Table 3). Other than this, there were no significant

Table 2. Comparison of the Zinc Normalized and Non-Normalized Groups of CD Patients 4 Weeks after ZAH Administration

\begin{tabular}{|c|c|c|c|}
\hline Variable & Zinc normalized group $(n=23)$ & Zinc non-normalized group $(n=9)$ & $P$-value \\
\hline Serum zinc levels before administration ( $\mu \mathrm{g} / \mathrm{dL})$ & 59 (46 to 74$)$ & 49 (31 to 69$)$ & 0.007 \\
\hline Changes in serum zinc levels ( $\mu \mathrm{g} / \mathrm{dL})$ & 41 (13 to 103$)$ & $10(-3$ to 17$)$ & $<0.001$ \\
\hline Dose of ZAH (mg/day) & $50(25$ to 150$)$ & $50(50$ to 100$)$ & 0.918 \\
\hline CDAI before administration & 152 (43 to 322 ) & 192 (48 to 546$)$ & 0.249 \\
\hline Changes in CDAI & $-23(-167$ to 55$)$ & $-36(-196$ to 16$)$ & 0.315 \\
\hline CRP before administration (mg/dL) & 0.15 (0.01 to 5.09) & 1.76 (0.04 to 12.82$)$ & 0.002 \\
\hline Changes in CRP (mg/dL) & $-0.01(-4.97$ to 0.19$)$ & $0.27(-12.80$ to 4.16$)$ & 0.294 \\
\hline Medical history (yr) & 18 (1 to 29$)$ & $15(1$ to 31$)$ & 0.346 \\
\hline Small intestinal lesion & $23(100)$ & 8 (88.9) & 0.104 \\
\hline Operation history & $15(65.2)$ & $6(66.7)$ & 0.938 \\
\hline No. of operations (times) & 1 (0 to 13$)$ & 1 (0 to 12$)$ & 0.821 \\
\hline Colostomy & $8(34.8)$ & $2(22.2)$ & 0.491 \\
\hline \multicolumn{4}{|l|}{ Concomitant medication } \\
\hline 5-ASA, SASP & 17 (73.9) & $6(66.7)$ & 0.682 \\
\hline Corticosteroid & $8(34.8)$ & 0 & 0.041 \\
\hline Immunomodulator & $16(69.6)$ & $3(33.3)$ & 0.061 \\
\hline Anti-TNF- $\alpha$ & $13(56.5)$ & $3(33.3)$ & 0.238 \\
\hline Anti-integrin & 0 & 0 & NS \\
\hline Anti-IL-12/-23 & $6(26.1)$ & $3(33.3)$ & 0.682 \\
\hline Enteral nutrition & $17(73.9)$ & $6(66.7)$ & 0.682 \\
\hline
\end{tabular}

Values are presented as median (range) or number (\%).

CD, Crohn's disease; ZAH, zinc acetate hydrate; CDAl, Crohn's Disease Activity Index; CRP, C-reactive protein; 5-ASA, 5-aminosalicylic acid; SASP, salazosulfapyridine; TNF, tumor necrosis factor; IL, interleukin; NS, not significant.

$P$-values were calculated using the chi-square test for categorical variables and Mann Whitney test for continuous variables. 
Table 3. Comparison of the Zinc Normalized and Non-Normalized Groups of CD Patients 20 Weeks after ZAH Administration

\begin{tabular}{|c|c|c|c|}
\hline Variable & Zinc normalized group $(n=13)$ & Zinc non-normalized group $(n=12)$ & $P$-value \\
\hline Serum zinc levels before administration $(\mu \mathrm{g} / \mathrm{dL})$ & 61 (47 to 71$)$ & 52 (31 to 73 ) & 0.100 \\
\hline Changes in serum zinc levels ( $\mu \mathrm{g} / \mathrm{dL}$ ) & $46(15$ to 127$)$ & 19 (1 to 74$)$ & 0.004 \\
\hline Dose of ZAH (mg/day) & 50 (25 to 150$)$ & 63 (50 to 100$)$ & 0.347 \\
\hline CDAl before administration & 177 (51 to 322$)$ & 189 (70 to 546$)$ & 0.602 \\
\hline Changes in CDAI & $-21(-137$ to 21$)$ & $-19(-183$ to 131$)$ & 0.948 \\
\hline CRP before administration (mg/dL) & 0.15 (0.02 to 5.36$)$ & 0.27 (0.02 to 12.82$)$ & 0.526 \\
\hline Changes in CRP (mg/dL) & $-0.01(-4.22$ to 0.21$)$ & $-0.06(-9.37$ to 5.38$)$ & 0.758 \\
\hline Medical history (yr) & 13 (1 to 31$)$ & 22 (1 to 32 ) & 0.477 \\
\hline Small intestinal lesion & $11(84.6)$ & $11(91.7)$ & 0.588 \\
\hline Operation history & $11(84.6)$ & $8(66.7)$ & 0.294 \\
\hline No. of operations (times) & 1 (0 to 13) & 2 (0 to 12$)$ & 0.776 \\
\hline Colostomy & $3(23.1)$ & $3(25.0)$ & 0.910 \\
\hline \multicolumn{4}{|l|}{ Concomitant medication } \\
\hline 5-ASA, SASP & $8(61.5)$ & $8(66.7)$ & 0.790 \\
\hline Corticosteroid & $2(15.4)$ & $2(16.7)$ & 0.930 \\
\hline Immunomodulator & $6(46.2)$ & $7(58.3)$ & 0.371 \\
\hline Anti-TNF- $\alpha$ & $9(69.2)$ & $5(41.6)$ & 0.165 \\
\hline Anti-integrin & 0 & 0 & NS \\
\hline Anti-IL-12/-23 & $2(18.2)$ & $4(33.3)$ & 0.294 \\
\hline Enteral nutrition & $5(38.4)$ & $10(83.3)$ & 0.022 \\
\hline
\end{tabular}

Values are presented as median (range) or number (\%).

CD, Crohn's disease; ZAH, zinc acetate hydrate; CDAl, Crohn's Disease Activity Index; CRP, C-reactive protein; 5-ASA, 5-aminosalicylic acid; SASP, salazosulfapyridine; TNF, tumor necrosis factor; IL, interleukin; NS, not significant.

$P$-values were calculated using the chi-square test for categorical variables and Mann Whitney test for continuous variables.

differences between the 2 groups for ZAH starting amount, CDAI score at the start of the administration, changes in CDAI score, changes in CRP, years of illness, presence or absence of small intestinal lesions, presence or absence of surgical history, presence or absence of an artificial anus, and the presence or absence of biologics.

\section{UC Patients}

\section{1) Patient Background}

The median age of the UC patients was 56 years (range, 28-87 years). This group consisted of 7 men (63.6\%) and 4 women (36.3\%) with a median duration of disease of 8 years (range, 0-47 years). The disease affected the left colon in 2 patients, while the remaining 9 patients experienced total colitis. The median partial Mayo score was 3 (range, 0-9), the median serum zinc level was $63 \mu \mathrm{g} / \mathrm{dL}$ (range, $46-74 \mu \mathrm{g} / \mathrm{dL}$ ), and the median ZAH dose was $100 \mathrm{mg} /$ day (range, 25-100 mg/day). Regarding concomitantly administered drugs, 10 patients
(91\%) received 5-aminosalicylic acid/salazosulfapyridine, 2 patients (18.1\%) received corticosteroids, 5 patients (45.4\%) received immunomodulators, 2 patients (18.1\%) received anti-TNF- $\alpha$ monoclonal antibodies, and 1 patient $(9.1 \%)$ received anti-integrin antibodies (Table 1). As symptoms associated with zinc deficiency, dysgeusia was observed in 3 cases and stomatitis was observed in 2 cases.

\section{2) Clinical Results}

There were 9 UC patients whose serum zinc levels were measured 4 weeks after starting ZAH administration. The median serum zinc levels before and after ZAH administration for 4 weeks improved significantly from 63.0 to $94.0 \mu \mathrm{g} / \mathrm{dL}(P=0.020)$, and serum zinc levels normalized in $88.9 \%$ of patients $(8 / 9)$. Furthermore, the median partial Mayo scores improved from $3(0-9)$ to $1(0-4)$, but no significant differences were observed $(P=0.125)$, and no significant difference was found in the analysis for each subscore (Fig. 5A and B, Supplementary Table 3). 

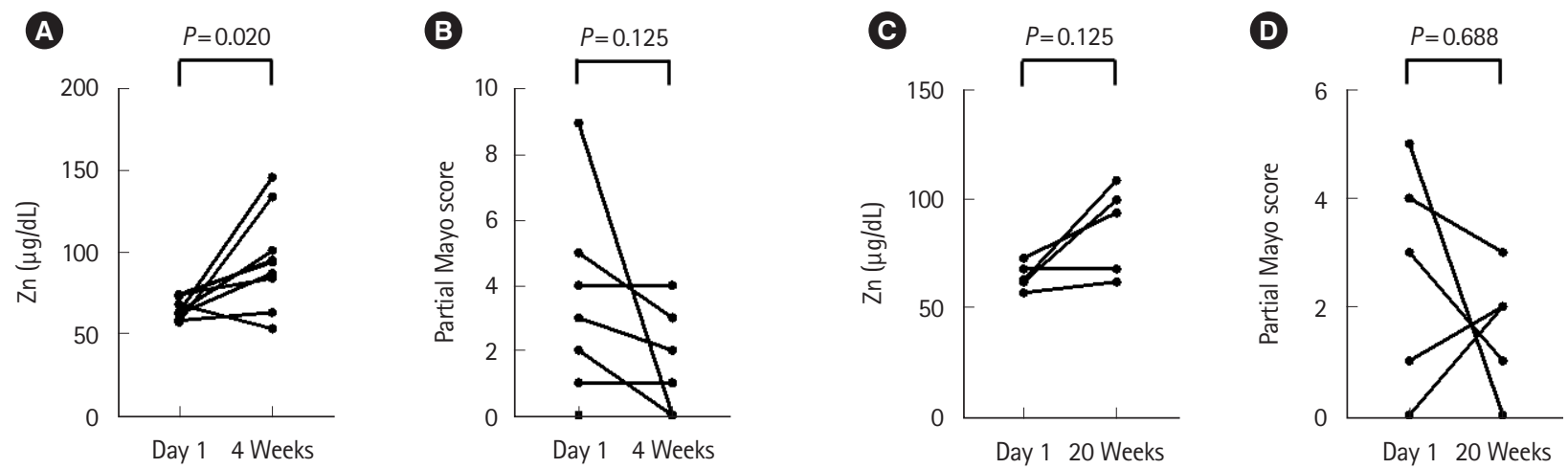

Fig. 5. Changes in serum zinc level and partial Mayo score in ulcerative colitis (UC) patients before zinc acetate hydrate (ZAH) administration and 4 weeks or 20 weeks after administration. (A, B) There were 9 UC patients whose serum zinc levels were measured 4 weeks after ZAH administration. Median serum zinc levels significantly improved from $63.0 \mu \mathrm{g} / \mathrm{dL}$ (57-74) to $94.0 \mu \mathrm{g} / \mathrm{dL}(53-146)(P=0.020)$. Median partial Mayo scores improved from $3(0-9)$ to $1(0-4)(P=0.125)$. (C, D) There were 5 UC patients whose serum zinc levels were measured 20 weeks after ZAH administration. Median serum zinc levels significantly improved from $63.0 \mu \mathrm{g} / \mathrm{dL}$ (57-73) to 94.0 $\mu \mathrm{g} / \mathrm{dL}$ (62109) $(P=0.125)$. Median partial Mayo scores before and after ZAH administration improved from $3(0-5)$ to $1(0-3)(P=0.688)$.

There were no notable changes in other blood sampling items 4 weeks after ZAH administration (Supplementary Table 4).

There were 5 UC patients whose serum zinc levels were measured 20 weeks after starting ZAH administration. The median serum zinc levels before and after ZAH administration for 20 weeks improved from 63.0 to $94.0 \mu \mathrm{g} / \mathrm{dL}(P=0.125)$, and serum zinc levels normalized in $60.0 \%(3 / 5)$ of patients. Furthermore, the median partial Mayo scores improved from 3 to 1 , but no significant differences were observed ( $P=0.688$ ), and no significant difference was observed in the analysis for each subscore (Fig. 5C and D, Supplementary Table 3).

There were no notable changes in other blood sampling items 20 weeks after ZAH administration (Supplementary Table 4).

The sample size for the UC patients was small, and no investigations could be conducted for the group in which treatment changes other than ZAH were not conducted during the observation period.

\section{Adverse Events}

Adverse effects were observed in 1 case and included abdominal discomfort and nausea. The symptoms disappeared immediately after drug administration was discontinued. There were a limited number of cases where serum copper levels were measured. However, the copper deficiency was observed in 1 out of 13 patients whose serum copper levels were measured 4 weeks after ZAH administration, and zero out of 14 patients whose serum copper levels were measured 20 weeks after ZAH administration.

\section{DISCUSSION}

In this observational study: (1) high improvements in zinc deficiency and disease activity were obtained in CD patients, regardless of 4 or 20 weeks after starting ZAH administration; (2) high improvements in zinc deficiency were obtained in UC patients 4 weeks after starting ZAH administration, whereas disease activity showed improving tendencies but no significant differences; (3) significant improvements in serum zinc level and disease activity were obtained in CD patients even for groups where there were no treatment changes other than ZAH during the observation period; (4) there were no clear factors which prevented zinc normalization in CD patients, but it was thought that high CRP and refractory small intestinal lesions which may require enteral nutrition were present; and (5) the adverse events of gastrointestinal disorders and copper deficiency were each identified in 1 patient.

Existing studies have pointed to micronutrient deficiencies in IBD patients. The frequency of these conditions tends to be high in those with high disease activity and those who have undergone many surgeries. ${ }^{5}$ Previous reports have found that the prevalence rate of zinc deficiency is nearly $40 \%$ in both CD and UC, and compared to the control group, the frequency of zinc deficiency in CD patients is high (the median serum zinc levels: $74.3 \pm 9.7 \mu \mathrm{g} / \mathrm{dL}$ in the CD group and $62.3 \pm 9.3 \mu \mathrm{g} / \mathrm{dL}$ in the control group; $P<0.001){ }^{10,11}$ Another study discovered that low zinc intake prior to UC onset is associated with the onset of the disease. ${ }^{20}$ Furthermore, IBD patients with zinc deficiency have higher risks of surgery, hospitalization, and complica- 
tions related to IBD. ${ }^{11}$ Thus, the frequency of zinc deficiency in IBD patients is high, and IBD patients experience disadvantages due to this deficiency.

Conversely, an animal experiment investigating the effectiveness of zinc replacement reported that administering zinc to mice with dextran sulfate sodium-induced enteritis contributed to the improvement in inflammation. ${ }^{21}$ Furthermore, a study of UC patients compared a group that received an intestinal injection of Polaprezinc ${ }^{\circledR}$ (a zinc-containing anti-ulcer agent) with another group that did not (the control group) and found that the Mayo and endoscopy scores (modified Matts' endoscopic classification) at 1 week significantly improved in the group for which the intestinal injection was performed. ${ }^{22}$ In CD patients, the permeability of the intestinal mucosa may be increased even when remission is achieved. On the other hand, Sturniolo et al..$^{23}$ performed a comparative examination of serum zinc levels and the permeability of intestinal mucosa, evaluated using the lactulose/mannitol ratio as an indicator. Serum zinc levels in CD patients before and after 8-week oral administration of zinc sulfate thrice daily were measured. This study found that serum zinc levels significantly increased following the administration of zinc sulfate, and the increase in serum zinc levels led to an improvement of the permeability of intestinal mucosa. ${ }^{23}$ However, it should be noted that the mean zinc level in the patients registered prior to the administration of zinc sulfate was $96.4 \mu \mathrm{g} / \mathrm{dL}$, which is within the normal range. Additionally, the patients were in a state of clinical remission (CDAI $<150$ was continuously maintained for at least 3 months prior to administration) when they received the zinc sulfate. To our knowledge, no research examining the usefulness of administering zinc to clinically active IBD patients with zinc deficiency has been published to date. Therefore, this research was conducted. As a result, this study indicates that the oral administration of zinc improved zinc deficiency in a large number of patients and improved disease activity in IBD patients.

As for the enrolled patient background, $85 \%$ of the patients in the $\mathrm{CD}$ group included small intestinal lesions. The main absorption sites for zinc are the duodenum and jejunum, and it is possible that inflammation in the small intestine caused impaired zinc absorption. ${ }^{24}$ In $62.5 \%$ of CD patients presented with zinc deficiency despite receiving enteral nutrition therapy containing zinc. The median CDAI before ZAH administration was 175 , which was mainly in patients with mild disease activity, but $80 \%$ of patients used Bio products. A comparison between the zinc normalized group and the non-normalized group showed no significant difference in the changes in CDAI and CRP before and after ZAH administration. However, there was a significant difference in the CRP before administration and the presence or absence of corticosteroid or enteral nutrition. The condition of the intestinal mucosa may be involved in the normalization of serum zinc level by ZAH administration, and it is necessary to evaluate the endoscopic activity of the intestinal mucosa before and after zinc administration in the future. Only $27.2 \%$ of UC patients were on Bio, and most were maintained in remission with 5ASA or immunomodulator.

It was difficult to examine patients at a fixed interval after starting ZAH administration since this was a retrospective study and the main subjects were outpatients. Therefore, this study set the time periods of 4-8 weeks after administration and 20-24 weeks after administration. The blood sampling data and disease activity at the points within these observation periods and the closest to 4 or 20 weeks after starting administration were used, and the changes in serum zinc level before and after ZAH administration and the accompanying changes in disease activity were evaluated.

Results showed that the serum zinc level and disease activity after ZAH administration significantly improved in the CD group compared to before ZAH administration. However, the possibility that improvements in disease activity due to other treatments may have influenced improvements in serum zinc levels could not be rejected. The same examinations were then conducted on groups where treatment changes did not occur other than administering ZAH within the observation period showed similar significant improvements in serum zinc level and disease activity in the CD group. Therefore, it is thought that the disease activity of CD may have improved from the normalization of serum zinc level. These are thought to be valuable results which support the active zinc replacement therapy for CD patients with zinc deficiency.

When the change in CDAI score was analyzed for each subscore, the number of liquid/soft stools in the past 1 week, the sum of 7 daily abdominal pain ratings, the sum of 7 daily wellbeing ratings, and bodyweight percent below standard weight were significantly improved. Originally, it has been reported that zinc deficiency causes chronic diarrhea and that zinc supplementation improves the permeability of the intestinal mucosa, which explains the reduction in the number of stools. ${ }^{5,22}$ For improvement of abdominal pain and body weight, endoscopic evaluation of the intestinal tract is still required. 
We analyzed whether there was a correlation between the degree of increase in serum zinc level and the degree of improvement in CDAI score or CRP, but no correlation was observed at any time of 4 weeks or 20 weeks after ZAH administration. In many cases, the serum zinc level after ZAH administration was $130 \mu \mathrm{g} / \mathrm{dL}$ or higher, and it was considered that increasing the serum zinc level above a certain level may not improve disease activity. On the other hand, a moderate correlation was demonstrated between serum zinc level and CDAI score or CRP before ZAH administration. To our knowledge, although there is a report on the correlation between urinary zinc level and CDAI score in patients with $\mathrm{CD}$, no report has been found that demonstrates a correlation between serum zinc level and CDAI score or CRP, and it is considered to be valuable data. ${ }^{25}$

ZAH was originally used as a therapeutic agent for Wilson's disease and has copper-chelating activity; therefore, patients receiving ZAH may develop copper deficiency. ${ }^{26,27}$ There were 13 and 14 patients in this study whose serum copper levels were measured during the course after 4 and 20 weeks, respectively. Of these, only 1 patient showed copper deficiency after 4 weeks, and ZAH could be used in a relatively safe manner. Measurements not only of serum zinc level but also serum copper level are thought to be essential during ZAH administration.

Other reported side effects primarily include gastrointestinal disorder, anemia, and skin disorders, but only 1 patient reported gastrointestinal disorders in this study, with symptoms rapidly improving after discontinuing ZAH administration.

There were several limitations to this study. First, the number of patients included was small. Second, there were very few cases in which endoscopy was performed before and after administration of ZAH, and the condition of the intestinal mucosa before and after administration of ZAH could not be evaluated. In addition, serum zinc level has a circadian rhythm, and it is known that it is also affected by diet and hormones such as insulin-like growth factor-1, free T4. ${ }^{28-30}$ However the patient background and sample collection status have not been unified. Therefore, in the future, it will be necessary to conduct a prospective study on zinc supplementation after unifying the patient background and blood collection time to evaluate the disease activity and the degree of inflammation of the intestinal mucosa. However, despite these limitations, this retrospective study is considered to be a valuable result in affirming aggressive zinc replacement therapy for CD patients with zinc deficiency.
In conclusion, our findings indicate that the administration of zinc formulations for IBD patients with zinc deficiency may normalize serum zinc levels, potentially improving disease activity, particularly in CD patients.

\section{ADDITIONAL INFORMATION}

\section{Funding Source}

The authors received no financial support for the research, authorship, and/or publication of this article.

\section{Conflict of Interest}

No potential conflict of interest relevant to this article was reported.

\section{Author Contribution}

Conceptualization: Sakurai K, Furukawa S, Katsurada T, Otagiri S, Yamanashi K, Onishi R, Yagisawa K, Nishimura H, Ito T, Maemoto A, Sakamoto N. Data curation: Sakurai K. Formal analysis: Sakurai K, Katsurada T, Otagiri S. Investigation: Sakurai K, Otagiri S. Methodology: Sakurai K, Furukawa S, Katsurada T, Otagiri S. Project administration: Sakurai K, Furukawa S, Otagiri S. Resources: Sakurai K, Katsurada T, Otagiri S. Software: Sakurai K, Otagiri S. Supervision: all authors. Validation: Sakurai K, Katsurada T, Otagiri S. Visualization: Sakurai K, Otagirip S. Writing - original draft: Sakuraip K. Writing - review \& editing: Sakurai K, Katsurada T, Otagiri S, Nagashima K, Onishi R. Approval of final manuscript: all authors.

\section{Others}

We thank the staff of Hokkaido University Hospital and Sapporo Higashi Tokushukai Hospital for their invaluable help with data collection.

\section{ORCID}

Sakurai K

Furukawa S

Katsurada T

Otagiri S

Yamanashi K

Nagashima K

Onishi R

Yagisawa K

Nishimura H

Ito $\mathrm{T}$

Maemoto A https://orcid.org/0000-0001-8178-8984 https://orcid.org/0000-0002-7054-5730 https://orcid.org/0000-0002-7400-2514 https://orcid.org/0000-0002-2209-9334 https://orcid.org/0000-0002-4680-8123 https://orcid.org/0000-0003-0528-0390 https://orcid.org/0000-0002-0353-4839 https://orcid.org/0000-0002-3316-5843 https://orcid.org/0000-0001-8458-5293 https://orcid.org/0000-0001-5228-4840 https://orcid.org/0000-0002-9182-8502 
Sakamoto N

https://orcid.org/0000-0003-0061-059X

\section{Supplementary Material}

Supplementary materials are available at the Intestinal Research website (https://www.irjournal.org).

\section{REFERENCES}

1. Mekhjian HS, Switz DM, Melnyk CS, Rankin GB, Brooks RK. Clinical features and natural history of Crohn's disease. Gastroenterology 1979;77(4 Pt 2):898-906.

2. Harries AD, Heatley RV. Nutritional disturbances in Crohn's disease. Postgrad Med J 1983;59:690-697.

3. Dawson AM. Nutritional disturbances in Crohn's disease. Br J Surg 1972;59:817-819.

4. Dawson AM. Nutritional disturbances in Crohn's disease. Proc R Soc Med 1971;64:166-170.

5. Hwang C, Ross V, Mahadevan U. Micronutrient deficiencies in inflammatory bowel disease: from A to zinc. Inflamm Bowel Dis 2012;18:1961-1981.

6. Lamb CA, Kennedy NA, Raine T, et al. British Society of Gastroenterology consensus guidelines on the management of inflammatory bowel disease in adults. Gut 2019;68(Suppl 3):s1s106.

7. Walsh CT, Sandstead HH, Prasad AS, Newberne PM, Fraker PJ. Zinc: health effects and research priorities for the 1990s. Environ Health Perspect 1994;102 Suppl 2(Suppl 2):5-46.

8. Filippi J, Al-Jaouni R, Wiroth JB, Hébuterne X, Schneider SM. Nutritional deficiencies in patients with Crohn's disease in remission. Inflamm Bowel Dis 2006;12:185-191.

9. Vagianos K, Bector S, McConnell J, Bernstein CN. Nutrition assessment of patients with inflammatory bowel disease. JPEN J Parenter Enteral Nutr 2007;31:311-319.

10. Solomons NW, Rosenberg IH, Sandstead HH, Vo-Khactu KP. Zinc deficiency in Crohn's disease. Digestion 1977;16:87-95.

11. Siva S, Rubin DT, Gulotta G, Wroblewski K, Pekow J. Zinc deficiency is associated with poor clinical outcomes in patients with inflammatory bowel disease. Inflamm Bowel Dis 2017; 23:152-157.

12. McClain C, Soutor C, Zieve L. Zinc deficiency: a complication of Crohn's disease. Gastroenterology 1980;78:272-279.

13. Sturniolo GC, Molokhia MM, Shields R, Turnberg LA. Zinc absorption in Crohn's disease. Gut 1980;21:387-391.

14. Naber TH, van den Hamer CJ, Baadenhuysen H, Jansen JB. The value of methods to determine zinc deficiency in patients with Crohn's disease. Scand J Gastroenterol 1998;33:514-523.
15. Tomita H. A proposal of the clinical standard value for diagnose of zinc deficiency by serum zinc value. Biomed Res Trace Elem 2008;19:22-24.

16. Matsuoka K, Kobayashi T, Ueno F, et al. Evidence-based clinical practice guidelines for inflammatory bowel disease. J Gastroenterol 2018;53:305-353.

17. Schroeder KW, Tremaine WJ, Ilstrup DM. Coated oral 5-aminosalicylic acid therapy for mildly to moderately active ulcerative colitis: a randomized study. N Engl J Med 1987;317: 1625-1629.

18. Best WR, Becktel JM, Singleton JW, Kern F Jr. Development of a Crohn's disease activity index. National Cooperative Crohn's Disease Study. Gastroenterology 1976;70:439-444.

19. Best WR, Becktel JM, Singleton JW. Rederived values of the eight coefficients of the Crohn's Disease Activity Index (CDAI). Gastroenterology 1979;77(4 Pt 2):843-846.

20. Kobayashi Y, Ohfuji S, Kondo K, et al. Association between dietary iron and zinc intake and development of ulcerative colitis: a case-control study in Japan. J Gastroenterol Hepatol 2019; 34:1703-1710.

21. Li J, Chen H, Wang B, et al. ZnO nanoparticles act as supportive therapy in DSS-induced ulcerative colitis in mice by maintaining gut homeostasis and activating Nrf2 signaling. Sci Rep 2017;7:43126.

22. Itagaki M, Saruta M, Saijo H, et al. Efficacy of zinc-carnosine chelate compound, Polaprezinc, enemas in patients with ulcerative colitis. Scand J Gastroenterol 2014;49:164-172.

23. Sturniolo GC, Di Leo V, Ferronato A, D'Odorico A, D'Incà R. Zinc supplementation tightens "leaky gut" in Crohn's disease. Inflamm Bowel Dis 2001;7:94-98.

24. Harzer G, Kauer H. Binding of zinc to casein. Am J Clin Nutr 1982;35:981-987.

25. Fleming CR, Huizenga KA, McCall JT, Gildea J, Dennis R. Zinc nutrition in Crohn's disease. Dig Dis Sci 1981;26:865-870.

26. Brewer GJ. Wilson's disease. In: Kasper DL, Braunwald E, Fauci AS, Hauser SL, Longo DL, Jameson JL, eds. Harrison's principles of internal medicine. 16th ed. New York: McGraw-Hill, 2005; 2313-2315

27. Illing AC, Shawki A, Cunningham CL, Mackenzie B. Substrate profile and metal-ion selectivity of human divalent metal-ion transporter-1. J Biol Chem 2012;287:30485-30496.

28. Kanabrocki EL, Sothern RB, Ryan MD, et al. Circadian characteristics of serum calcium, magnesium and eight trace elements and of their metallo-moieties in urine of healthy middle-aged men. Clin Ter 2008;159:329-346.

29. McMillan EM, Rowe DJ. Clinical significance of diurnal varia- 
tion in the estimation of plasma zinc. Clin Exp Dermatol 1982;7:629-632.

30. Food and Nutrition Board, Institute of Medicine. Zinc. In: Dietary reference intakes for vitamin A, vitamin $\mathrm{K}$, arsenic, bo- ron, chromium, copper, iodine, iron, manganese, molybdenum, nickel, silicon, vanadium, and zinc. Washington, DC: National Academy Press, 2001:442-501. 
See "Effectiveness of administering zinc acetate hydrate to patients with inflammatory bowel disease and zinc deficiency: a retrospective observational two-center study" on page 78-89.

Supplementary Table 1. Changes in the CDAI Score for Each Subscore before and after ZAH Administration in CD Patients

\begin{tabular}{|c|c|c|c|c|c|c|}
\hline \multirow{2}{*}{ Variable } & \multicolumn{3}{|c|}{ At 4 week } & \multicolumn{3}{|c|}{ At 20 week } \\
\hline & Day 1 & 4 Weeks & $P$-value & Day 1 & 20 Weeks & $P$-value \\
\hline No. of liquid/soft stools, $1 \mathrm{wk}$ & $56(0-210)$ & $46(0-140)$ & 0.018 & $60(0-210)$ & $58(0-210)$ & 0.558 \\
\hline Sum of 7 daily abdominal pain ratings & $0(0-70)$ & $0(0-70)$ & 0.007 & $0(0-70)$ & $0(0-70)$ & 0.133 \\
\hline Sum of 7 daily ratings, well being & $28(0-147)$ & $0(0-112)$ & $<0.001$ & $14(0-147)$ & $0(119)$ & 0.047 \\
\hline No. of other groups of symptoms/findings & $0(0-40)$ & $0(0-20)$ & 0.125 & $0(0-40)$ & $0(0-20)$ & 0.125 \\
\hline Arthritis/arthralgias & $1(3.1)$ & $1(3.1)$ & NS & 0 & 0 & NS \\
\hline Iritis/uveitis & 0 & 0 & NS & 0 & 0 & NS \\
\hline $\begin{array}{l}\text { Erythema nodosum, pyoderma gangrenosum, or } \\
\text { aphthous stomatitis }\end{array}$ & $1(3.1)$ & 0 & 0.314 & $1(4)$ & $1(4)$ & NS \\
\hline Anal fissure, fistula, or abscess & $3(9.4)$ & $1(3.1)$ & 0.302 & $3(12)$ & 0 & 0.074 \\
\hline Other fistula & $1(3.1)$ & 0 & 0.314 & $1(4)$ & 0 & 0.312 \\
\hline Fever/temperature $>100^{\circ} \mathrm{F} / 37.8^{\circ} \mathrm{C}$ & $1(3.1)$ & 0 & 0.314 & $1(4)$ & 0 & 0.312 \\
\hline Taking opiate antidiarrheal agent & $0(0-30)$ & $0(0-30)$ & 0.500 & $0(0-30)$ & $0(0-30)$ & 0.500 \\
\hline Abdominal mass & $0(0-20)$ & $0(0-0)$ & NS & $0(0-20)$ & $0(0-0)$ & NS \\
\hline Hematocrit & $46(0-110)$ & $44(0-128)$ & 0.161 & $44(17-110)$ & $44(8-104)$ & 0.677 \\
\hline Bodyweight percent below standard weight & $12(0-31)$ & $11.5(0-29)$ & 0.041 & $13(0-27)$ & $13(0-25)$ & 0.066 \\
\hline
\end{tabular}

Values are presented as median (range) or number (\%). The values calculated for each score are listed. For the following items, the number of people (\%) with each symptom is listed. Arthritis/arthralgias, iritis/uveitis, erythema nodosum, pyoderma gangrenosum, or aphthous stomatitis, anal fissure, fistula, or abscess, other fistulae, fever/temperature $>100^{\circ} \mathrm{F} / 37.8^{\circ} \mathrm{C}$.

CDAl, Crohn's Disease Activity Index; ZAH, zinc acetate hydrate; CD, Crohn's disease; NS, not significant.

$P$-values were calculated using the chi-squared test for categorical variables and the Wilcoxon matched-pairs signed-rank test for continuous variables. 
Supplementary Table 2. Laboratory Data before and after ZAH Administration in CD Patients

\begin{tabular}{|c|c|c|c|c|c|c|}
\hline \multirow{2}{*}{ Variable } & \multicolumn{3}{|c|}{ At 4 weeks } & \multicolumn{3}{|c|}{ At 20 weeks } \\
\hline & Day 1 & 4 Weeks & $P$-value & Day 1 & 20 Weeks & $P$-value \\
\hline WBC $(/ \mu \mathrm{L})$ & $6,475(3,350-13,950)$ & $6,850(4,110-13,750)$ & 0.367 & $6,430(3,110-13,950)$ & $6,190(2,680-16,200)$ & 0.634 \\
\hline $\mathrm{Hb}(\mathrm{g} / \mathrm{dL})$ & $12.6(8.5-15.1)$ & $12.4(8.0-16.1)$ & 0.702 & $12.5(8.5-15.1)$ & $12.3(9.2-15.5)$ & 0.593 \\
\hline $\mathrm{PLT}\left(\times 10^{4} / \mu \mathrm{L}\right)$ & $28.4(11.7-42.7)$ & $27.9(10.1-49.5)$ & 0.965 & $27.8(11.7-41.1)$ & $28.6(13.7-43.2)$ & 0.249 \\
\hline $\mathrm{CRP}(\mathrm{mg} / \mathrm{dL})$ & $0.31(0.01-12.82)$ & $0.12(0.01-9.61)$ & 0.213 & $0.18(0.02-12.82)$ & $0.25(0.01-5.44)$ & 0.182 \\
\hline ALB (g/dL) & $3.4(1.8-4.8)$ & $3.6(1.9-4.4)$ & 0.141 & $3.5(1.8-4.8)$ & $3.7(2.9-4.6)$ & 0.105 \\
\hline
\end{tabular}

Values are presented as median (range).

ZAH, zinc acetate hydrate; $C D$, Crohn's disease; WBC, white blood cell; Hb, hemoglobin; PLT, platelet; CRP, C-reactive protein; ALB, albumin.

$P$-values were calculated using the Wilcoxon matched-pairs signed-rank test for continuous variables. 
Supplementary Table 3. Changes in the Partial Mayo Score for Each Subscore before and after ZAH Administration in UC Patients

\begin{tabular}{|c|c|c|c|c|c|c|}
\hline \multirow{2}{*}{ Variable } & \multicolumn{3}{|c|}{ At 4 weeks } & \multicolumn{3}{|c|}{ At 20 weeks } \\
\hline & Day 1 & 4 Weeks & $P$-value & Day 1 & 20 Weeks & $P$-value \\
\hline Stool frequency & $1(0-3)$ & $1(0-2)$ & 0.750 & $1(0-2)$ & $1(0-2)$ & NS \\
\hline Physician's global assessment & $2(0-3)$ & $0(0-2)$ & 0.375 & $0(0-2)$ & $0(0-1)$ & 0.750 \\
\hline
\end{tabular}

Values are presented as median (range).

$\mathrm{ZAH}$, zinc acetate hydrate; UC, ulcerative colitis; NS, not significant.

$P$-values were calculated using the Wilcoxon matched-pairs signed-rank test for continuous variables. 
Supplementary Table 4. Laboratory Data before and after ZAH Administration in UC Patients

\begin{tabular}{|c|c|c|c|c|c|c|}
\hline \multirow{2}{*}{ Variable } & \multicolumn{3}{|c|}{ At 4 weeks } & \multicolumn{3}{|c|}{ At 20 weeks } \\
\hline & Day 1 & 4 Weeks & $P$-value & Day 1 & 20 Weeks & $P$-value \\
\hline WBC $(/ \mu \mathrm{L})$ & $5,390(2,660-10,890)$ & $5,900(3,860-9,330)$ & 0.820 & $5,390(2,660-6,690)$ & $4,510(3,330-6,000)$ & 0.813 \\
\hline $\mathrm{Hb}(\mathrm{g} / \mathrm{dL})$ & 12. $6(8.5-14.6)$ & $12.4(10.4-14.4)$ & 0.488 & $11.7(10.2-13.4)$ & $12.1(10.5-13.4)$ & 0.875 \\
\hline $\mathrm{PLT}\left(\times 10^{4} / \mu \mathrm{L}\right)$ & $20.6(9.4-35.2)$ & $21.6(16.7-34.4)$ & 0.289 & $19.9(11.0-24.4)$ & $21.2(17.3-24.9)$ & 0.188 \\
\hline $\mathrm{CRP}(\mathrm{mg} / \mathrm{dL})$ & $0.70(0.02-7.27)$ & $0.33(0.01-1.07)$ & 0.055 & $0.63(0.17-1.48)$ & $0.09(0.03-0.37)$ & 0.063 \\
\hline ALB (g/dL) & $3.7(3.4-4.3)$ & $3.9(3.4-4.3)$ & 0.496 & $3.7(3.5-4.1)$ & $3.9(3.7-4.0)$ & 0.625 \\
\hline
\end{tabular}

Values are presented as median (range).

ZAH, zinc acetate hydrate; UC, ulcerative colitis; WBC, white blood cell; Hb, hemoglobin; PLT, platelet; CRP, C-reactive protein; ALB, albumin.

$P$-values were calculated using the Wilcoxon matched-pairs signed-rank test for continuous variables. 